\title{
Targeting cascade screening in familial hypercholesterolaemia
}

Around $60 \%$ of individuals with a clinical diagnosis of familial hypercholesterolaemia might have a polygenic cause for the disorder, report Talmud and colleagues. Restricting genetic testing of relatives of index patients to those with a monogenic cause of the disease $(\sim 40 \%)$ could improve the cost effectiveness of a cascade screening strategy, the researchers suggest.

Familial hypercholesterolaemia is an autosomal-dominant disorder in which the level of LDL cholesterol is considerably raised, which increases the risk of early coronary heart disease. The disorder is caused by a mutation in one of three known genes. Importantly, individuals with familial hypercholesterolaemia and affected relatives can be treated with statins, which improves life expectancy.

"The current study was designed to investigate whether individuals who inherit many small-effect LDLcholesterol-raising alleles might have received a clinical diagnosis of familial hypercholesterolaemia," explains senior researcher Steve Humphries from University College London, UK, "which would influence the efficacy of any cascade screening programme since the odds of finding relatives with grossly elevated LDLcholesterol levels in such cases would be considerably less than the expected 50\%."

The researchers genotyped British patients with familial hypercholesterolaemia for 12 common alleles that raise the level of LDL-cholesterol. The cohort included 319 patients who carried a known familial hypercholesterolaemia mutation, 321 of whom were mutation-negative, and 3,020 healthy control individuals. The investigators determined a weighted LDLcholesterol-raising gene score for each individual. Patients who were mutationnegative had a significantly higher mean weighted LDL-cholesterol-raising gene score than control participants. This finding suggests that a considerable proportion of these patients had a polygenic cause of the disorder. Analyses in patients with familial hypercholesterolaemia from Belgium produced similar findings.

John Kastelein from the University of Amsterdam, the Netherlands, who was not involved in the study, points out that the findings of the study could stem from the patient population used.
"The frequency of mutations in familial hypercholesterolaemia is a consequence of clinical sampling. In our hands, hit rates are well above $80 \%$ in children and adults."

Kastelein adds that cascade screening has resulted in the identification of 27,000 patients with familial hypercholesterolaemia in the Netherlands. "We disagree that cascade screening by cholesterol measurement would not make sense in polygenic hypercholesterolaemia: finding individuals with elevated LDLcholesterol levels leads to statin use and the prevention of heart attacks," he comments.

\section{Carol Wilson}

This article is modified from the original in Nature Reviews Endocrinology (doi:10.1038/ nrendo.2013.53).

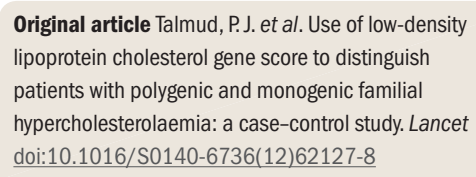

